Research Paper

\title{
Melatonin alleviates oxidative stress-inhibited osteogenesis of human bone marrow-derived mesenchymal stem cells through AMPK activation
}

\author{
Sooho Lee ${ }^{1}$, Nhu Huynh Le ${ }^{1,2}$, and Dongchul Kang ${ }^{1,2}$ \\ 1. Ilsong Institute of Life Science, Hallym University, Anyang, Gyeonggi-do 14066, Republic of Korea. \\ 2. Department of Biomedical Gerontology, Hallym University Graduate School, Chuncheon, Gangwon-do 24252, Republic of Korea. \\ $\bowtie$ Corresponding author: Dongchul Kang dckang@hallym.ac.kr \\ (c) Ivyspring International Publisher. This is an open access article distributed under the terms of the Creative Commons Attribution (CC BY-NC) license \\ (https://creativecommons.org/licenses/by-nc/4.0/). See http://ivyspring.com/terms for full terms and conditions.
}

Received: 2018.03.27; Accepted: 2018.06.08; Published: 2018.06.23

\begin{abstract}
Oxidative stress plays an important role in the pathogenesis of aging-related osteoporosis through the increased bone resorption or reduced bone formation. Melatonin, which can exert beneficial actions through antioxidant, anti-inflammatory, and bone-preserving effects, shows promise in preventing oxidative stress-inhibited osteogenesis. However, specific mechanisms by which melatonin rescues oxidative stress-inhibited osteogenesis of human mesenchymal stem cells (MSCs) have not been fully elucidated yet. We therefore investigated whether activation of AMPK by melatonin regulates the antagonistic crosstalk between oxidative stress and osteogenic differentiation in human MSCs. Melatonin treatment significantly enhanced osteogenic differentiation of human MSCs through activation of AMPK and upregulation of FOXO3a and RUNX2 which were known as master transcription factors responsible for the mechanistic link between oxidative stress and osteogenic phenotype. Osteogenic differentiation determined by calcium deposition was significantly increased by melatonin treatment against oxidative stress. In addition, melatonin treatment reconstituted activation of AMPK and expression of FOXO3a and RUNX2 inhibited by oxidative stress. Overall, these results demonstrate that melatonin enhances osteogenic differentiation of human MSCs and restores oxidative stress-inhibited osteogenesis through AMPK activation in human MSCs, suggesting that activation of AMPK by melatonin may represent a promising new therapeutic strategy for treating metabolic bone diseases such as osteoporosis.
\end{abstract}

Key words: melatonin, oxidative stress, mesenchymal stem cells, osteogenesis, AMPK, osteoporosis

\section{Introduction}

Osteoporosis is the most common bone metabolic disease that is characterized by decreased bone mass and structural deterioration of bone tissue, leading to an increased risk of bone fracture at the different skeletal sites such as spine, hip and wrist [1]. The incidence of osteoporosis is closely related to aging in both women and men, which is associated with oxidative stress [2]. Recently, much attention has been paid to the adverse effects of oxidative stress on bone formation $[3,4]$. Oxidative stress shifts a balance in bone remodeling toward increased bone resorption by osteoclasts and decreased bone formation by osteoblasts, which can eventually result in accelerated osteoporosis [5]. Oxidative stress has been shown to inhibit osteogenic potential of MSCs and promotes apoptosis of mature osteoblasts [6, 7]. Furthermore, several studies showed that oxidative stress could interfere with multiple cellular events that induced MSC differentiation, including Wnt/beta-catenin and FOXO signaling pathways [8-10].

Human MSCs are multipotent adult progenitor cells that have a capacity for self-renewal and can differentiate into specialized cell types such as osteocytes, adipocytes and chondrocytes [11]. MSCs 
have been easily isolated and expanded without severe functional damages from various sources, including bone marrow, adipose tissue, umbilical cord blood and Wharton's jelly which harbor a stem cell population [12]. MSCs that manifested the immunomodulatory properties produced several cytokines and growth factors in order to create supportive microenvironment for themselves in host tissue [13]. Moreover, several studies have demonstrated that MSCs can be used as ideal candidates for tissue regeneration due to their capability to replace damaged tissue at sites of injury in vivo [14].

Melatonin, $\mathrm{N}$-acetyl-5-methoxytryptamine, is a tryptophan-derived hormone secreted by the pineal gland in the brain. Melatonin has drawn considerable therapeutic interest for various disorders as a consequence of its multiple biological functions including control of circadian rhythms, tumor inhibition, antioxidant activity, and immunomodulatory properties [15]. Recently, melatonin has been demonstrated to exert protective effects against ischemia/reperfusion injury in vitro and in vivo via inhibition of oxidative stress, inflammation and apoptosis, supporting that melatonin has antioxidant properties with strong cytoprotective activities [16-18].

Melatonin has also an influence on skeleton formation and development through regulating the balance between bone resorption by osteoclasts and bone formation by osteoblasts. Melatonin at pharmacological concentrations suppressed the osteoclast differentiation of mouse bone marrow-derived monocytes in a dose-dependent manner via attenuation of intracellular ROS and inhibition of the NF-kB signaling pathway $[19,20]$. Treatment with high doses of melatonin (up to 50 $\mathrm{mg} / \mathrm{kg}$ ) caused an inhibition of bone resorption and an increase in bone mass in mice [21]. In contrast to the inhibitory effect of melatonin on bone resorption by osteoclast, stimulatory effects of melatonin on bone formation have been reported previously [22-24]. Melatonin has also been shown to play an important role in directing the differentiation of MSCs towards specific lineages [25, 26]. Taken together, these results suggest that melatonin shifts bone remodeling toward bone formation over bone resorption by osteoclasts. However, the mechanism for melatonin to promote osteogenic differentiation of MSCs has not been fully understood. Furthermore, whether antioxidant activity of melatonin can also restore osteogenic potential of MSC inhibited by oxidative stress and its protective mechanism against the oxidative stress remains to be determined.

AMP-activated protein kinase (AMPK), a highly conserved serine/threonine kinase, exists as a heterotrimeric complex of a catalytic a subunit and two regulatory $\beta$ and $\gamma$ subunits [27]. AMPK plays a critical role as a metabolic sensor in maintaining both cellular and whole-body energy homeostasis by modulating glucose and lipid metabolism, as well as by facilitating appropriate adaptive responses to ATP-consuming conditions such as ischemia/ reperfusion, hypoxia, oxidative stress, and exercise [28]. Moreover, AMPK has emerged as a potential therapeutic target for the treatments of a variety of diseases, including obesity, type 2 diabetes, cardiovascular diseases, and other metabolic diseases $[29$, 30]. Indeed, it has been documented that pharmacological activation of AMPK has been shown to provide cardioprotection against myocardial ischemia/reperfusion injury in animal models of type 2 diabetes [31, 32]. Recent studies have also shown that AMPK activation could positively regulates bone homeostasis through enhancement of the osteogenic potential of MSCs [33-37]. However, there are no studies examining whether AMPK activation is involved in the effect of melatonin on the osteogenic potential of MSCs.

In the present study, considering the relevance of oxidative stress as a risk factor in the development of osteoporosis, we investigated the effect of melatonin on osteogenic differentiation per se and oxidative stress-inhibited osteogenic differentiation of human MSCs and the underlying mechanisms. We demonstrated that melatonin enhanced osteogenic potential of MSCs and effectively antagonized the deleterious effects of oxidative stress on osteoblast differentiation of the MSCs through AMPK activation.

\section{Materials and methods}

\section{Reagents and antibodies}

The cell culture plates and flasks were purchased from SPL Life Sciences (Pocheon, South Korea). a-Minimum essential medium (a-MEM) was purchased from Gibco (Grand Island, NY, USA). Antibiotics (10,000 units/mL penicillin and 10,000 $\mathrm{\mu g} / \mathrm{mL}$ streptomycin) were purchased from Hyclone (Logan, UT, USA). Fetal bovine serum (FBS) was purchased from Welgene (Daegu, South Korea). Compound C was purchased from Calbiochem (Darmstadt, Germany). Protease inhibitor cocktail tablets were purchased from Thermo Fisher Scientific (Waltham, MA, USA). The primary antibodies against phospho-AMPKa (Thr172) and RUNX2 were purchased from Cell Signaling Technology (Danvers, MA, USA), and antibodies against AMPKa1/2, FOXO3a, and $\beta$-actin were purchased from Santa Cruz Biotechnology (Santa Cruz, CA, USA). The 
horseradish peroxidase (HRP)-conjugated secondary antibodies were purchased from Invitrogen (Carlsbad, CA, USA). All other reagents were obtained from Sigma-Aldrich (St. Louis, MO, USA) unless otherwise specified.

\section{Cell culture}

Human bone marrow-derived MSCs were purchased from ScienCell Research Laboratories (Cat. No. 7500; Carlsbad, CA, USA) and maintained in a growth medium consisting of a-MEM supplemented with 16.5\% FBS and antibiotics (100 units/mL penicillin and $100 \mu \mathrm{g} / \mathrm{mL}$ streptomycin) at $37^{\circ} \mathrm{C}$ in a humidified atmosphere of 5\% $\mathrm{CO}_{2}$ and $95 \%$ air. Cells between passages 3 and 10 were used for all experiments.

\section{Cell viability assay}

Cell viability was assessed by the methylthiazolyldiphenyl-tetrazolium bromide (MTT) assay. Briefly, cells were seeded in 96-well microplates at $8 \times 10^{3}$ cells/well and incubated at specified conditions for an indicated time period. Medium was aspirated and then cells were incubated with $100 \mu \mathrm{l}$ MTT solution ( $5 \mathrm{mg} / \mathrm{ml}$ MTT in PBS) for 4 $h$. After the MTT formazan crystals were dissolved in $100 \mu \mathrm{l}$ of lysis buffer containing $10 \%$ SDS in $0.01 \mathrm{~N}$ $\mathrm{HCl}$, the absorbance was measured at $570 \mathrm{~nm}$ using a Multiskan ${ }^{\mathrm{TM}}$ GO microplate reader (Thermo Fisher Scientific).

\section{Osteoblast differentiation and $\mathrm{H}_{2} \mathrm{O}_{2}$ treatment}

For osteogenic differentiation, human MSCs were plated at density of $3 \times 10^{5}$ cells/well in 6-well plates or $1 \times 10^{4}$ cells/well in 96-well plates and incubated in growth medium until confluent. At that point, the growth medium was replaced with osteogenic differentiation medium (ODM) consisting of a-MEM supplemented with 10\% FBS, $100 \mathrm{nM}$ dexamethasone, $10 \mathrm{mM} \beta$-glycerophosphate, $50 \mu \mathrm{M}$ ascorbic-2-phosphate, 100 units/mL penicillin and $100 \mu \mathrm{g} / \mathrm{mL}$ streptomycin. Fresh ODM was replenished twice per week. For the rescue experiment, human MSCs were pre-exposed to 100 $\mu \mathrm{M} \mathrm{H}_{2} \mathrm{O}_{2}$ (diluted in growth medium) for $2 \mathrm{~h}$. The cells were washed twice with fresh growth medium and followed by incubation in ODM with or without indicated concentrations of melatonin.

\section{Alkaline phosphatase (ALP) activity assay}

ALP activity as an early marker of osteogenic differentiation was assessed at day 4. Cells were washed twice with PBS and then lysed with protein lysis buffer (50 mM Tris- $\mathrm{HCl} \mathrm{pH}$ 7.4, $150 \mathrm{mM} \mathrm{NaCl}, 1$ mM EDTA, and 1\% NP-40). ALP activity was determined colorimetrically by incubating the protein lysates with substrate $p$-nitrophenyl phosphate in 96-well plates at $37^{\circ} \mathrm{C}$ for $30 \mathrm{~min}$. The absorbance was measured at $405 \mathrm{~nm}$ and normalized against the corresponding protein amounts. The values were expressed as fold change relative to undifferentiated cells.

\section{Alizarin Red S staining}

Osteogenic differentiation of human MSCs was assessed by Alizarin Red S staining for the presence of calcium deposits. Briefly, the cells were washed twice with PBS, fixed with $4 \%$ formaldehyde for $30 \mathrm{~min}$ at room temperature, rinsed with distilled water, and then stained with $2 \%(\mathrm{w} / \mathrm{v})$ Alizarin Red S dissolved in distilled water ( $\mathrm{pH}$ 4.2; adjusted with 10\% ammonium hydroxide) for $20 \mathrm{~min}$. Cells were then washed extensively with distilled water and examined for mineralization. After imaging, the dye was eluted with $10 \%(\mathrm{w} / \mathrm{v})$ cetylpyridinium chloride monohydrate in $10 \mathrm{mM}$ sodium phosphate $(\mathrm{pH} 7.0)$ for $1 \mathrm{~h}$ at room temperature, and the absorbance was measured at $570 \mathrm{~nm}$ using a Multiskan ${ }^{\mathrm{TM}}$ GO microplate reader (Thermo Fisher Scientific).

\section{Western blot analysis}

Cells were washed twice with PBS and lysed in RIPA lysis buffer (50 mM Tris- $\mathrm{HCl}$ pH 7.4, $150 \mathrm{mM}$ $\mathrm{NaCl}, 1 \mathrm{mM}$ EDTA, 1\% NP-40, 0.1\% SDS, 0.5\% sodium deoxycholate, $5 \mathrm{mM}$ sodium fluoride, $2 \mathrm{mM}$ sodium orthovanadate, $1 \mathrm{mM}$ PMSF, and protease inhibitor cocktail). Protein concentrations in the supernatant were determined using a BCA protein assay kit (Thermo Fisher Scientific). Equal amounts of total protein $(25 \mu \mathrm{g})$ were separated on $10 \%$ SDS-PAGE and transferred onto Hybond-ECL nitrocellulose membranes (Amersham, Arlington Heights, IL, USA). The membranes were blocked with Tris-buffered saline-Tween 20 (TBS-T: $10 \mathrm{mM}$ Tris- $\mathrm{HCl} \mathrm{pH}$ 7.6, $150 \mathrm{mM} \mathrm{NaCl}$, and 0.1\% Tween 20) containing 5\% nonfat dry milk and incubated with primary antibodies diluted in blocking buffer overnight at $4^{\circ} \mathrm{C}$. The membranes were washed three times with TBS-T and then incubated with appropriate HRP-conjugated secondary antibodies for $1 \mathrm{~h}$ at room temperature. The blots were visualized using ECL detection reagents (Advansta, Menlo Park, CA, USA).

\section{Statistical analysis}

All data were expressed as the mean \pm standard error of the mean (SEM). Differences between groups were examined for statistical significance using Student's $t$-test. The difference was considered to be significant if $P<0.05$. 
A

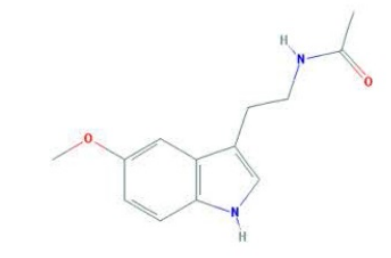

$\mathrm{N}$-acetyl-5-methoxytryptamine

(MF: $\mathrm{C}_{13} \mathrm{H}_{16} \mathrm{~N}_{2} \mathrm{O}_{2}, \mathrm{MW}: 232.28$ )
B

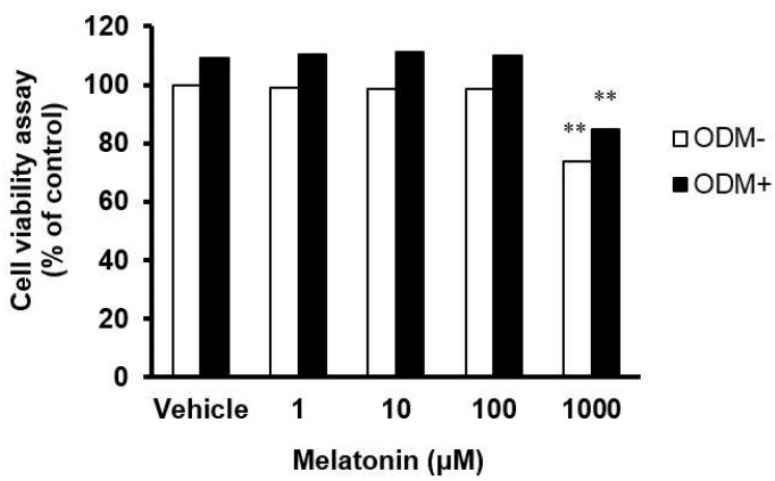

Figure 1. Effects of melatonin on cell viability. (A) Chemical structure of melatonin (from National Center of Biotechnology Information, PubChem CID: 896). (B) Human MSCs were seeded in 96-well plates at a density of $8 \times 10^{3}$ cells/well and then treated with or without ODM containing indicated concentrations of melatonin for 3 days. Cell viability was determined using the MTT assay. Data are represented as mean \pm SEM of three individual experiments $(n=3)$. Statistical significance was determined using Student's $t$-test $\left({ }^{*} p<0.05 ;{ }^{* *} p<0.01\right.$; compared with untreated control).

\section{Results}

\section{Effects of melatonin on cell viability}

Cell viability of human MSCs treated with 1,10 , 100 , and 1,000 $\mu \mathrm{M}$ melatonin was determined using the MTT assay (Fig. 1A and B). There was no toxicity at the concentrations of 1,10 , and $100 \mu \mathrm{M}$, but the cell viability was decreased by toxicity at the concentration of $1,000 \mu \mathrm{M}$, regardless of the type of medium used (basal or osteogenic), suggesting a dose-dependent effect of melatonin on the viability of human MSCs. Therefore, the subsequent experiments were carried out at concentrations of 1, 10, and 100 $\mu \mathrm{M}$.

\section{Melatonin stimulates osteoblast differentiation of human MSCs}

We next investigated the effects of melatonin on both early and late stages of osteoblast differentiation process. Human MSCs were treated with ODM and melatonin at different doses during the first 4 days. ALP activity assay was performed to assess the effect of melatonin on the early stage of osteoblast differentiation (Fig. 2A). ALP activity was higher in the osteogenic medium-treated group compared with the control group, and was significantly increased further by treatment with melatonin in a dose-dependent manner. Interestingly, melatonin alone also promotes osteoblast differentiation of human MSCs in a dose-dependent manner, as judged by increasing their ALP activity. The degree of calcium deposition was also detected by Alizarin Red $S$ staining (Fig. 2B and C). When melatonin $(100 \mu \mathrm{M})$ was treated for 14 days, the degree of calcium deposition was markedly increased when compared with human MSCs treated with ODM alone. These results indicate that melatonin can exert a synergistic effect on osteoblast differentiation and may be used as a pro-osteogenic agent in stem cell based-therapy.

\section{Melatonin significantly enhances AMPK activation during osteoblast differentiation of human MSCs}

To confirm whether the melatonin promotes osteoblast differentiation through AMPK activation, human MSCs were treated with vehicle or indicated concentrations of melatonin for $24 \mathrm{~h}$ with ODM. AMPK phosphorylation and the expression levels of its downstream effectors, FOXO3a and RUNX2 that were closely associated with the osteoblast differentiation, were detected by Western blot analysis. Interestingly, the activating phosphorylation of AMPK and the protein expression of FOXO3a and RUNX2 were significantly increased by melatonin in a dose-dependent manner (Fig. 3A), which were suppressed by co-treatment of compound C, a synthetic AMPK inhibitor (Fig. 3B). These findings indicate that AMPK activation is responsible for enhanced osteoblast differentiation of human MSCs by melatonin.

\section{Melatonin restores oxidative stress-inhibited osteoblast differentiation of human MSCs by activating AMPK}

Since AMPK activation has been reported to protect cells from oxidative stress [38, 39], we tested the effect of AMPK activation by melatonin on oxidative stress-inhibited osteoblast differentiation. Human MSCs were pretreated with $100 \mathrm{\mu M} \mathrm{H}_{2} \mathrm{O}_{2}$ for 2 $\mathrm{h}$, which has no detectable adverse effect on cell viability [40], and then immediately treated with ODM. After 21 days of incubation was completed, 
AMPK activation and osteoblast differentiation were detected by Western blot analysis and Alizarin Red S staining, respectively. As shown in Fig. 4, $\mathrm{H}_{2} \mathrm{O}_{2}$ treatment markedly reduced not only the osteogenic potential of human MSCs, but also AMPK activation and protein expression of its downstream effectors, FOXO3a and RUNX2. However, these effects were

A

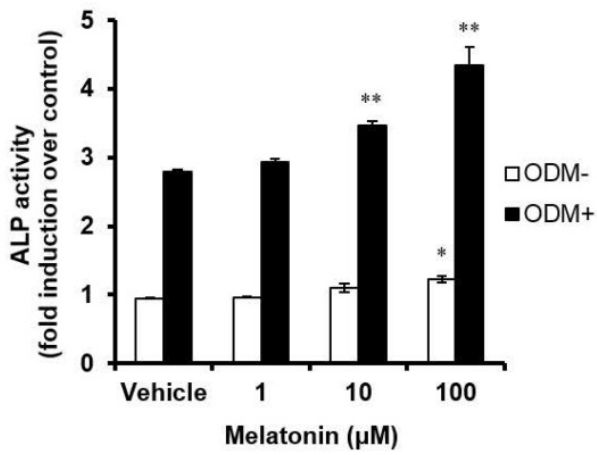

C

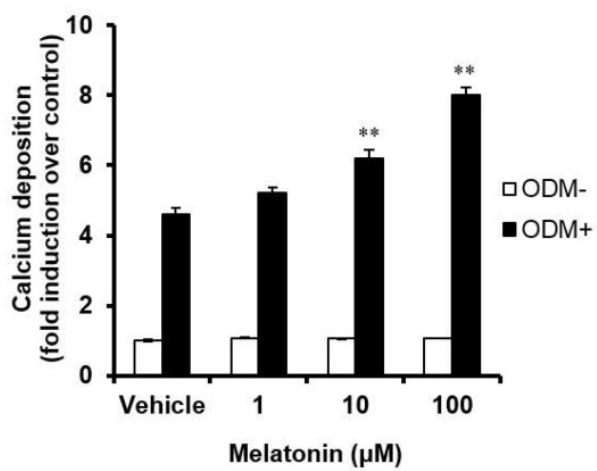

reversed by melatonin treatment in a dose-dependent manner. Taken together, our findings indicate that AMPK plays an important role in the regulation of pro-osteogenic signals in human MSCs and that melatonin alleviates the oxidative stress-induced inhibition of osteoblast differentiation of human MSCs through AMPK activation.

B

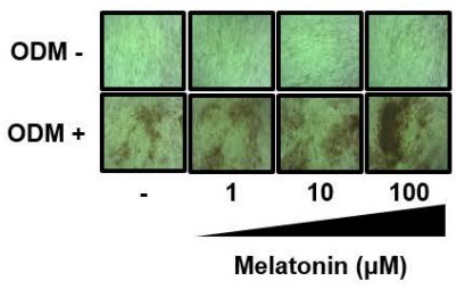

Figure 2. Melatonin stimulates osteoblast differentiation in human MSCs. (A) Human MSCs were seeded in 96 -well plates at a density of $8 \times 10^{3}$ cells/well and then treated with or without ODM containing indicated concentrations of melatonin for first 4 days. (B) The cells were treated with or without ODM containing indicated concentrations of melatonin for 14 days, followed by Alizarin Red S staining and visualized by phase-contrast microscopy at a final magnification of $200 X$. (C) The mineralized layers were dissolved and quantified using a microplate reader at $570 \mathrm{~nm}$. Data are represented as mean \pm SEM of three individual experiments $(n=3)$. Statistical significance was determined using Student's t-test ( $p<0.05 ; * * 0.01$; compared with untreated control).

A

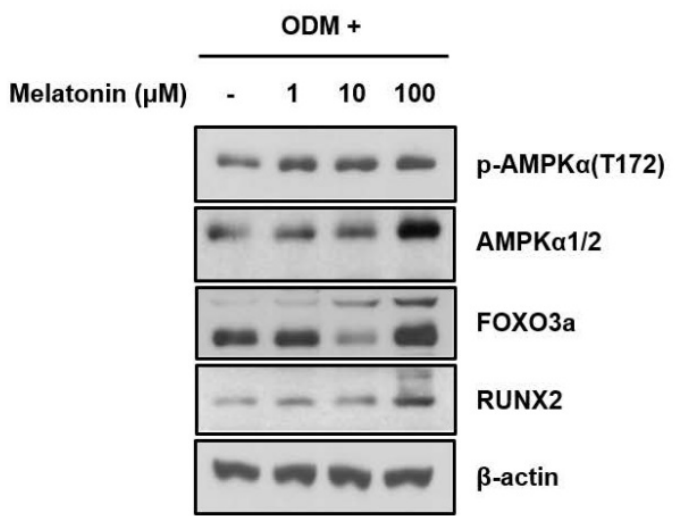

B

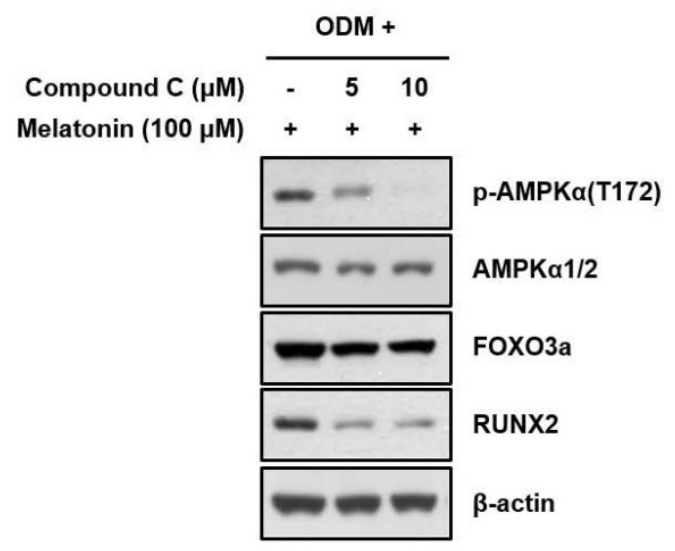

Figure 3. Melatonin significantly enhances AMPK activation during osteoblast differentiation of human MSCs. (A) Human MSCs were treated with the indicated concentrations of melatonin for $24 \mathrm{~h}$ under ODM. (B) Cell were treated with the indicated concentrations of melatonin (100 $\mu \mathrm{M})$ and compound $\mathrm{C}$ for $24 \mathrm{~h}$ under ODM. Western blot analysis was performed with the specified antibodies as described in Materials and Methods. Representative data from multiple experiments are shown. 
A

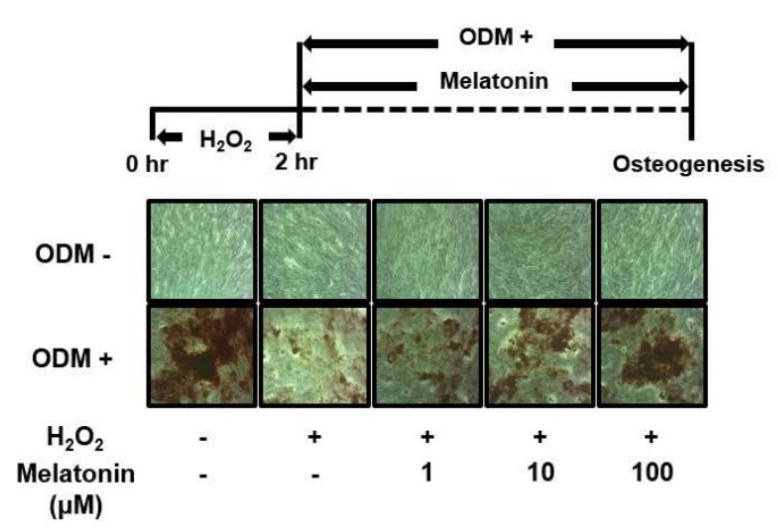

B

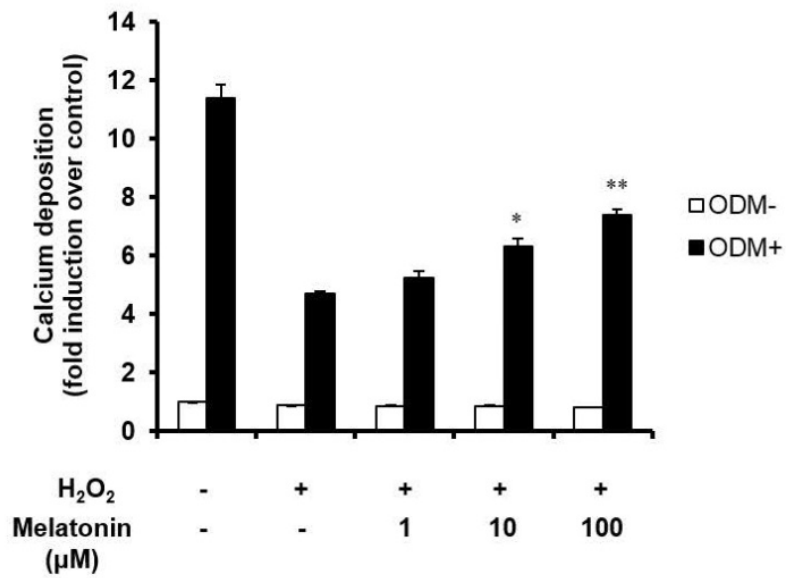

C
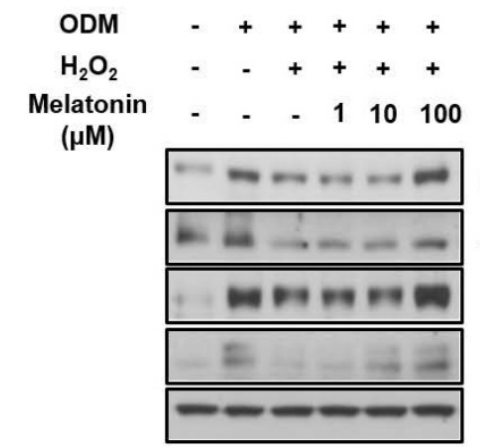

p-AMPKa(T172)

AMPK $\alpha 1 / 2$

FOXO3a

RUNX2

$\beta$-actin

Figure 4. Melatonin restores oxidative stress-inhibited osteoblast differentiation of human MSCs by activating AMPK signaling. (A) Human MSCs were treated with $100 \mu \mathrm{M} \mathrm{H}_{2} \mathrm{O}_{2}$ and incubated in ODM with the indicated concentrations of melatonin for 21 days. The calcium deposition of human MSCs was assessed by Alizarin Red S staining. (B) The mineralized layers were dissolved and quantified using a microplate reader at $570 \mathrm{~nm}$. (C) Total proteins were subjected to Western blot analysis using the specified antibodies. Data are represented as mean \pm SEM of three individual experiments $(n=3)$. Statistical significance was determined using Student's $t$-test $\left({ }^{*} p<0.05\right.$ and $p<0.01$ in contrast to the group treated with $\mathrm{H}_{2} \mathrm{O}_{2}$ alone).

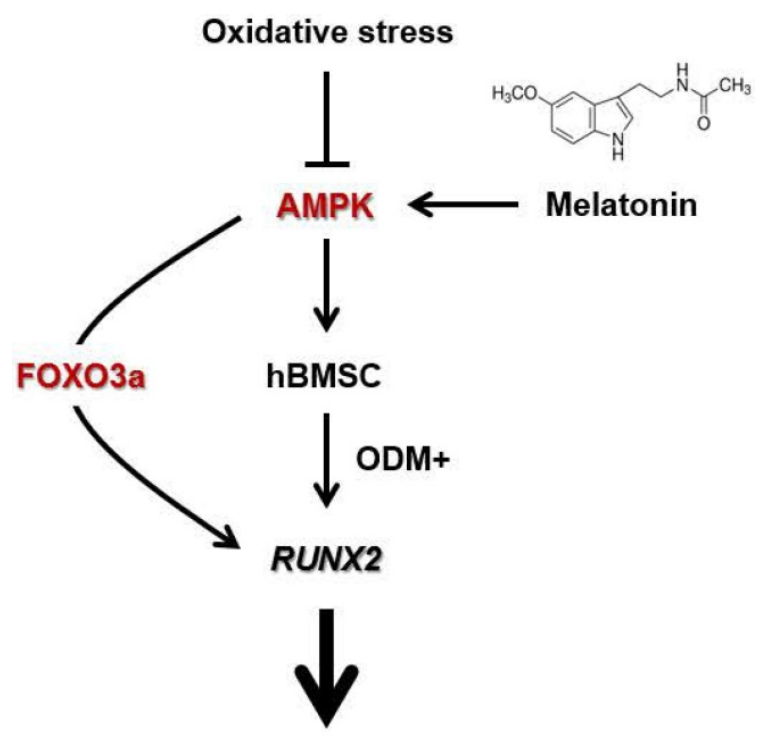

Osteogenesis

Figure 5. A scheme showing the mechanism by which melatonin protects human MSCs against oxidative stress-inhibited osteogenesis. Oxidative stress inhibits osteoblast differentiation of human MSCs by decreasing AMPK signaling. However, melatonin supplement alleviates oxidative stress-inhibited osteogenesis by restoring the in vitro differentiation potential of human MSCs through activation of AMPK-FOXO3a-RUNX2 axis. The proposed scheme suggests a therapeutic potential of melatonin in MSC-based bone regeneration and repair.

\section{Discussion}

Osteoporosis is a major public health problem throughout the world [1, 2]. Accumulating studies indicate that oxidative stress is responsible for age-related bone loss and might play an important role in development of osteoporosis in both men and women [3, 5]. Not only could oxidative stress influence osteoblast proliferation and survival, but also has a direct impact on its differentiation [6]. In the present study, we demonstrated that melatonin, widely known as an antioxidant, showed significant protective potential against oxidative stress-induced inhibition of osteoblast differentiation in human MSCs.

Age-related skeletal changes, including decreased osteoblast number as well as decreased bone mass and strength, are closely associated with increased oxidative stress [4]. Additionally, oxidative stress-induced premature cellular senescence was found to impair osteogenic differentiation potential of human and mouse MSCs [41, 42]. Consistent with previous reports [43], we found that oxidative stress induced the reduction in cellular ALP activity and 
subsequently diminished calcium deposition in parallel with a significant decrease in both FOXO3a (the key transcription factor regulating oxidative stress-induced cellular response) and RUNX2 (the key transcriptional factor initiating osteogenesis) protein levels during human MSC osteogenesis (Fig. 4), suggesting that oxidative stress, at least in part, could contribute to the dysfunction of tissue-specific stem/progenitor cells and that targeting oxidative stress may improve multi-lineage differentiation potential and clinical utilities of MSCs.

Osteoblast differentiation from MSCs is a well-orchestrated process and regulated by multiple signaling pathways $[44,45]$. Among them is AMPK which has been emerged as a master regulator of whole-body energy homeostasis by coordinating various aspects of metabolism, such as food intake, energy expenditure, insulin secretion, hepatic glucose production, and glucose/fatty acid metabolism in skeletal muscle and adipose tissue [27, 28]. Therefore, functional disturbances of AMPK could have been linked with a wide range of cellular malfunctions and diseases [30]. Regarding the importance of AMPK signaling pathway in bone metabolism, it has recently been reported that AMPK regulates bone formation and bone mass both in vitro and in vivo [46-48]. In addition, AMPK plays an essential role in directing human MSC differentiation and fate specification [34, $49,50]$. Several studies have also demonstrated the protective effect of AMPK against oxidative stress in different cell types [38, 51-53]. In this study, we found that treatment of melatonin at $100 \mu \mathrm{M}$ by itself could enhance osteogenic potential of human MSCs by activation of AMPK (Fig. 2). Furthermore, we demonstrated that AMPK activation by melatonin was accompanied with the increased protein expression levels of FOXO3a and RUNX2 (Fig. 3) as markers of oxidative stress/antioxidant defense and osteogenic potential, respectively. Therefore, activation of AMPK by melatonin is of critical importance not only in stimulation of the osteogenic potential of human MSCs, but also in protection of the potential against oxidative stress. AMPK activation is capable of eliciting the crosstalk between oxidative damage and bone formation.

FOXO proteins, characterized by a common winged-helix DNA binding domain called the forkhead box, are an evolutionarily conserved subfamily of transcription factors which play critical roles in a wide variety of biological processes including tumor suppression, regulation of energy metabolism and development in several tissues [54]. FOXO proteins are mainly regulated by phosphorylation-dependent nuclear-cytoplasmic shuttling [55]. From the viewpoint of their significance in bone formation [9, 10, 56, 57], FOXO-dependent defense mechanism against oxidative damage provides an implement for cellular adaptation to cope with oxidative free radicals generated as normal byproducts of aerobic metabolism of osteoblasts and is thereby essential for maintaining the bone mass homeostasis. FOXO3a, one of the four mammalian FOXO family members (FOXO1, FOXO3, FOXO4 and FOXO6), also promotes osteogenesis by stimulating RUNX2 gene expression which is a key transcription factor functionally related to the lineage determination and differentiation of MSCs [58]. Moreover, AMPK is required to directly or indirectly mediate the FOXO3a transcriptional activity in oxidative stress response [59-62]. We found that melatonin promotes osteogenesis in human MSCs by activating the AMPK signaling pathway, which is accompanied by increased FOXO3a and RUNX2 protein levels. In addition, melatonin alleviates oxidative stress-inhibited osteogenesis of human MSCs by activating AMPK and subsequently up-regulating of FOXO3a and RUNX2 protein levels. Taken together, these results can constitute a mechanism in which activation of AMPK by melatonin mediates upregulation of FOXO3a and RUNX2, which, in turn, stimulates osteogenic potential of human MSCs per se or alleviates oxidative stress-induced inhibition of the potential (Fig. 5). It is worth noticing that melatonin inhibits bone resorption by osteoclast through its antioxidant capacity [20]. Therefore, these results may provide new insights for the development of novel therapeutic strategies for combating bone metabolic diseases like osteoporosis.

\section{Conclusion}

In this work, we demonstrate that melatonin stimulates the osteogenesis of human MSCs by activating the AMPK pathway. We also found that melatonin enhanced the restoration of oxidative stress-impaired osteogenesis of human MSCs in a dose-dependent manner in vitro. The molecular mechanism by which melatonin exerts the protective effect on human MSCs against oxidative stress is at least in part associated with an increased levels of endogenous FOXO3a and RUNX2 proteins through the activation of AMPK pathway. Our work suggests that activation of AMPK signaling by melatonin supplements may represent a new therapeutic strategy for treating metabolic bone diseases.

\section{Abbreviations}

MSCs: mesenchymal stem cells; AMPK: AMP-activated protein kinase; a-MEM: a-minimum essential medium; FBS: fetal bovine serum; HRP: 
horseradish peroxidase; MTT: methylthiazolyldiphenyl-tetrazolium bromide; ODM: osteogenic differentiation medium; ALP: alkaline phosphatase; TBS-T: Tris-buffered saline-Tween 20; SEM: standard error of the mean.

\section{Acknowledgements}

This work was supported by the Basic Science Research Program through the National Research Foundation of Korea (NRF) funded by the Ministry of Education, Science and Technology (No. 2011-0025290 \& No. 2017R1D1A3B03035436).

\section{Author contributions}

SL and DK participated in the conception and design of research; SL and NHL performed the experiments; SL and DK analyzed the data; SL and DK interpreted results of experiments; SL and DK prepared figures; SL and DK drafted manuscript; SL and DK edited and revised manuscript; SL, NHL, and DK approved final version of manuscript.

\section{Competing Interests}

The authors have declared that no competing interest exists.

\section{References}

1. Eastell R, O'Neill TW, Hofbauer LC, Langdahl B, Reid IR, Gold DT, Cummings SR. Postmenopausal osteoporosis. Nat Rev Dis Primers. 2016; 2:16069.

2. Manolagas SC. From estrogen-centric to aging and oxidative stress: a revised perspective of the pathogenesis of osteoporosis. Endocr Rev. 2010; 31(3):266-300.

3. Callaway DA, Jiang JX. Reactive oxygen species and oxidative stress in osteoclastogenesis, skeletal aging and bone diseases. J Bone Miner Metab. 2015; 33(4):359-370

4. Goettsch C, Babelova A, Trummer O, Erben RG, Rauner M, Rammelt S, Weissmann N, Weinberger V, Benkhoff S, Kampschulte M, et al. NADPH oxidase 4 limits bone mass by promoting osteoclastogenesis. J Clin Invest. 2013; 123(11):4731-4738.

5. Hendrickx G, Boudin E, Van Hul W. A look behind the scenes: the risk and pathogenesis of primary osteoporosis. Nat Rev Rheumatol. 2015; 11(8):462-474.

6. Atashi F, Modarressi A, Pepper MS. The role of reactive oxygen species in mesenchymal stem cell adipogenic and osteogenic differentiation: a review. Stem Cells Dev, 2015; 24(10):1150-1163.

7. Vono R, Jover Garcia E, Spinetti G, Madeddu P. Oxidative Stress in Mesenchymal Stem Cell Senescence: Regulation by Coding and Noncoding RNAs. Antioxid Redox Signal. 2017; [Epub ahead of print].

8. Almeida M, Han L, Martin-Millan M, O'Brien CA, Manolagas SC. Oxidative stress antagonizes Wnt signaling in osteoblast precursors by diverting beta-catenin from $\mathrm{T}$ cell factor- to forkhead box O-mediated transcription. J Biol Chem. 2007: 282(37) $27298-27305$.

9. Ambrogini E, Almeida M, Martin-Millan M, Paik JH, Depinho RA, Han L, Goellner J, Weinstein RS, Jilka RL, O'Brien CA, et al. FoxO-mediated defense against oxidative stress in osteoblasts is indispensable for skeletal homeostasis in mice. Cell Metab. 2010; 11(2):136-146.

10. Rached MT, Kode A, Xu L, Yoshikawa Y, Paik JH, Depinho RA, Kousteni S. FoxO1 is a positive regulator of bone formation by favoring protein synthesis and resistance to oxidative stress in osteoblasts. Cell Metab. 2010; 11(2):147-160

11. Chen Q, Shou P, Zheng C, Jiang M, Cao G, Yang Q, Cao J, Xie N, Velletri T, Zhang $\mathrm{X}$, et al. Fate decision of mesenchymal stem cells: adipocytes or osteoblasts? Cell Death Differ. 2016; 23(7):1128-1139.

12. Nombela-Arrieta C, Ritz J, Silberstein LE. The elusive nature and function of mesenchymal stem cells. Nat Rev Mol Cell Biol. 2011; 12(2):126-131.

13. Loebel C, Burdick JA. Engineering Stem and Stromal Cell Therapies for Musculoskeletal Tissue Repair. Cell Stem Cell. 2018; 22(3):325-339.

14. Samsonraj RM, Raghunath M, Nurcombe V, Hui JH, van Wijnen AJ, Cool SM. Concise Review: Multifaceted Characterization of Human Mesenchymal Stem
Cells for Use in Regenerative Medicine. Stem Cells Transl Med. 2017; 6(12):2173-2185

15. Lee MS, Yin TC, Sung PH, Chiang JY, Sun CK, Yip HK. Melatonin enhances survival and preserves functional integrity of stem cells: A review. J Pineal Res. 2017; 62(2):e12372.

16. Zhai M, Li B, Duan W, Jing L, Zhang B, Zhang M, Yu L, Liu Z, Yu B, Ren K, et al. Melatonin ameliorates myocardial ischemia reperfusion injury through SIRT3-dependent regulation of oxidative stress and apoptosis. J Pineal Res. 2017; 63(2):e12419.

17. Zhang S, Chen S, Li Y, Liu Y. Melatonin as a promising agent of regulating stem cell biology and its application in disease therapy. Pharmacol Res. 2017; $117: 252-260$

18. Zhou H, Ma Q, Zhu P, Ren J, Reiter RJ, Chen Y. Protective role of melatonin in cardiac ischemia-reperfusion injury: From pathogenesis to targeted therapy. J Pineal Res. 2018; 64(3):e12471.

19. Ping Z, Wang Z, Shi J, Wang L, Guo X, Zhou W, Hu X, Wu X, Liu Y, Zhang W, et al. Inhibitory effects of melatonin on titanium particle-induced inflammatory bone resorption and osteoclastogenesis via suppression of NF-kappaB signaling. Acta Biomater. 2017; 62:362-371.

20. Zhou L, Chen X, Yan J, Li M, Liu T, Zhu C, Pan G, Guo Q, Yang H, Pei M, et al. Melatonin at pharmacological concentrations suppresses osteoclastogenesis via the attenuation of intracellular ROS. Osteoporos Int. 2017; 28(12):3325-3337.

21. Koyama H, Nakade O, Takada $\mathrm{Y}$, Kaku $\mathrm{T}$, Lau KH. Melatonin at pharmacologic doses increases bone mass by suppressing resorption through down-regulation of the RANKL-mediated osteoclast formation and activation. J Bone Miner Res. 2002; 17(7):1219-1229.

22. Park KH, Kang JW, Lee EM, Kim JS, Rhee YH, Kim M, Jeong SJ, Park YG, Kim $\mathrm{SH}$. Melatonin promotes osteoblastic differentiation through the BMP/ERK/Wnt signaling pathways. J Pineal Res. 2011; 51(2):187-194.

23. Zhang L, Su P, Xu C, Chen C, Liang A, Du K, Peng Y, Huang D. Melatonin inhibits adipogenesis and enhances osteogenesis of human mesenchymal stem cells by suppressing PPARgamma expression and enhancing Runx2 expression. J Pineal Res. 2010; 49(4):364-372.

24. Zhou L, Chen X, Liu T, Gong Y, Chen S, Pan G, Cui W, Luo ZP, Pei M, Yang H, et al. Melatonin reverses $\mathrm{H}_{2} \mathrm{O}_{2}$-induced premature senescence in mesenchymal stem cells via the SIRT1-dependent pathway. J Pineal Res. 2015; 59(2):190-205.

25. Amstrup AK, Sikjaer T, Mosekilde L, Rejnmark L. Melatonin and the skeleton. Osteoporos Int. 2013; 24(12):2919-2927.

26. Luchetti F, Canonico B, Bartolini D, Arcangeletti M, Ciffolilli S, Murdolo G, Piroddi M, Papa S, Reiter RJ, Galli F. Melatonin regulates mesenchymal stem cell differentiation: a review. J Pineal Res. 2014; 56(4):382-397.

27. Garcia D, Shaw RJ. AMPK: Mechanisms of Cellular Energy Sensing and Restoration of Metabolic Balance. Mol Cell. 2017; 66(6):789-800.

28. Lin SC, Hardie DG. AMPK: Sensing Glucose as well as Cellular Energy Status. Cell Metab. 2018; 27(2):299-313.

29. Day EA, Ford RJ, Steinberg GR. AMPK as a Therapeutic Target for Treating Metabolic Diseases. Trends Endocrinol Metab. 2017; 28(8):545-560.

30. Olivier S, Foretz M, Viollet B. Promise and challenges for direct small molecule AMPK activators. Biochem Pharmacol. 2018; 153:147-158.

31. Chang W, Li K, Guan F, Yao F, Yu Y, Zhang M, Hatch GM, Chen L. Berberine Pretreatment Confers Cardioprotection Against Ischemia-Reperfusion Injury in a Rat Model of Type 2 Diabetes. J Cardiovasc Pharmacol Ther. 2016; 21(5):486-494.

32. Yi W, Sun $Y$, Gao E, Wei X, Lau WB, Zheng $\mathrm{O}$ Wang $Y$, Yuan $Y$, Wang X, Tao $\mathrm{L}$, et al. Reduced cardioprotective action of adiponectin in high-fat diet-induced type II diabetic mice and its underlying mechanisms. Antioxid Redox Signal. 2011; 15(7):1779-1788

33. Barbagallo I, Vanella A, Peterson SJ, Kim DH, Tibullo D, Giallongo C, Vanella L, Parrinello N, Palumbo GA, Di Raimondo F, et al. Overexpression of heme oxygenase-1 increases human osteoblast stem cell differentiation. J Bone Miner Metab. 2010; 28(3):276-288

34. Kim EK, Lim S, Park JM, Seo JK, Kim JH, Kim KT, Ryu SH, Suh PG. Human mesenchymal stem cell differentiation to the osteogenic or adipogenic lineage is regulated by AMP-activated protein kinase. J Cell Physiol. 2012; 227(4):1680-1687

35. Molinuevo MS, Schurman L, McCarthy AD, Cortizo AM, Tolosa MJ, Gangoiti MV, Arnol V, Sedlinsky C. Effect of metformin on bone marrow progenitor cell differentiation: in vivo and in vitro studies. J Bone Miner Res. 2010; 25(2):211-221.

36. Pantovic A, Krstic A, Janjetovic K, Kocic J, Harhaji-Trajkovic L, Bugarski D, Trajkovic V. Coordinated time-dependent modulation of AMPK/Akt/mTOR signaling and autophagy controls osteogenic differentiation of human mesenchymal stem cells. Bone. 2013; 52(1):524-531.

37. Wang P, Ma T, Guo D, Hu K, Shu Y, Xu HHK, Schneider A. Metformin induces osteoblastic differentiation of human induced pluripotent stem cell-derived mesenchymal stem cells. J Tissue Eng Regen Med. 2018; 12(2):437-446.

38. Han X, Tai H, Wang X, Wang Z, Zhou J, Wei X, Ding Y, Gong H, Mo C, Zhang $\mathrm{J}$, et al. AMPK activation protects cells from oxidative stress-induced senescence via autophagic flux restoration and intracellular $\mathrm{NAD}(+)$ elevation. Aging Cell. 2016; 15(3):416-427.

39. Yang $\mathrm{H}$, Feng A, Lin S, Yu L, Lin X, Yan X, Lu X, Zhang C. Fibroblast growth factor-21 prevents diabetic cardiomyopathy via AMPK-mediated 
antioxidation and lipid-lowering effects in the heart. Cell Death Dis. 2018; 9(2):227.

40. Wang D, Wang Y, Xu S, Wang F, Wang B, Han K, Sun D, Li L. Epigallocatechin-3-gallate Protects against Hydrogen Peroxide-Induced Inhibition of Osteogenic Differentiation of Human Bone Marrow-Derived Mesenchymal Stem Cells. Stem Cells Int. 2016; 2016:7532798.

41. Ho PJ, Yen ML, Tang BC, Chen CT, Yen BL. $\mathrm{H}_{2} \mathrm{O}_{2}$ accumulation mediates differentiation capacity alteration, but not proliferative decline, in senescent human fetal mesenchymal stem cells. Antioxid Redox Signal. 2013; 18(15):1895-1905.

42. Turinetto V, Vitale E, Giachino C. Senescence in Human Mesenchymal Stem Cells: Functional Changes and Implications in Stem Cell-Based Therapy. Int J Mol Sci. 2016; 17(7):1164.

43. Huang Q, Gao B, Jie Q, Wei BY, Fan J, Zhang HY, Zhang JK, Li XJ, Shi J, Luo $\mathrm{ZJ}$, et al. Ginsenoside-Rb2 displays anti-osteoporosis effects through reducing oxidative damage and bone-resorbing cytokines during osteogenesis. Bone. 2014; 66:306-314

44. Jeyabalan J, Shah M, Viollet B, Chenu C. AMP-activated protein kinase pathway and bone metabolism. J Endocrinol. 2012; 212(3):277-290.

45. Lin GL, Hankenson KD. Integration of BMP, Wnt, and notch signaling pathways in osteoblast differentiation. J Cell Biochem. 2011; 112(12):3491-3501.

46. Kang H, Viollet B, Wu D. Genetic deletion of catalytic subunits of AMP-activated protein kinase increases osteoclasts and reduces bone mass in young adult mice. J Biol Chem. 2013; 288(17):12187-12196.

47. Shah M, Kola B, Bataveljic A, Arnett TR, Viollet B, Saxon L, Korbonits M, Chenu C. AMP-activated protein kinase (AMPK) activation regulates in vitro bone formation and bone mass. Bone. 2010; 47(2):309-319.

48. Xi G, Rosen CI, Clemmons DR. IGF-I and IGFBP-2 Stimulate AMPK Activation and Autophagy, Which Are Required for Osteoblast Differentiation. Endocrinology. 2016; 157(1):268-281.

49. Lee S, Cho HY, Bui HT, Kang D. The osteogenic or adipogenic lineage commitment of human mesenchymal stem cells is determined by protein kinase C delta. BMC Cell Biol. 2014; 15:42.

50. Tatapudy S, Aloisio F, Barber D, Nystul T. Cell fate decisions: emerging roles for metabolic signals and cell morphology. EMBO Rep. 2017; 18(12):2105-2118.

51. Gopoju R, Panangipalli S, Kotamraju S. Metformin treatment prevents SREBP2-mediated cholesterol uptake and improves lipid homeostasis during oxidative stress-induced atherosclerosis. Free Radic Biol Med. 2018; 118:85-97.

52. Guo X, Jiang Q, Tuccitto A, Chan D, Alqawlaq S, Won GJ, Sivak JM. The AMPK-PGC-1alpha signaling axis regulates the astrocyte glutathione system to protect against oxidative and metabolic injury. Neurobiol Dis. 2018; 113:59-69.

53. Wang C, Mao C, Lou Y, Xu J, Wang Q, Zhang Z, Tang Q, Zhang X, Xu H, Feng $Y$. Monotropein promotes angiogenesis and inhibits oxidative stress-induced autophagy in endothelial progenitor cells to accelerate wound healing. J Cell Mol Med. 2018; 22(3):1583-1600.

54. van der Horst A, Burgering BM. Stressing the role of FoxO proteins in lifespan and disease. Nat Rev Mol Cell Biol. 2007; 8(6):440-450.

55. Tzivion G, Dobson M, Ramakrishnan G. FoxO transcription factors; Regulation by AKT and 14-3-3 proteins. Biochim Biophys Acta. 2011; 1813(11):1938-1945.

56. Bartell SM, Kim HN, Ambrogini E, Han L, Iyer S, Serra Ucer S, Rabinovitch P, Jilka RL, Weinstein RS, Zhao $\mathrm{H}$, et al. FoxO proteins restrain osteoclastogenesis and bone resorption by attenuating $\mathrm{H}_{2} \mathrm{O}_{2}$ accumulation. Nat Commun. 2014; 5:3773.

57. Rached MT, Kode A, Silva BC, Jung DY, Gray S, Ong H, Paik JH, DePinho RA, Kim JK, Karsenty G, et al. FoxO1 expression in osteoblasts regulates glucose homeostasis through regulation of osteocalcin in mice. J Clin Invest. 2010; 120(1):357-368.

58. Tseng PC, Hou SM, Chen RJ, Peng HW, Hsieh CF, Kuo ML, Yen ML. Resveratrol promotes osteogenesis of human mesenchymal stem cells by upregulating RUNX2 gene expression via the SIRT1/FOXO3A axis. J Bone Miner Res. 2011; 26(10):2552-2563.

59. Greer EL, Banko MR, Brunet A. AMP-activated protein kinase and FoxO transcription factors in dietary restriction-induced longevity. Ann N Y Acad Sci. 2009; 1170:688-692.

60. Hong YA, Lim JH, Kim MY, Kim Y, Park HS, Kim HW, Choi BS, Chang YS, Kim HW, Kim TY, et al. Extracellular Superoxide Dismutase Attenuates Renal Oxidative Stress Through the Activation of Adenosine Monophosphate-Activated Protein Kinase in Diabetic Nephropathy. Antioxid Redox Signal. 2018; 28(17):1543-1561.

61. Ido Y, Duranton A, Lan F, Weikel KA, Breton L, Ruderman NB. Resveratrol prevents oxidative stress-induced senescence and proliferative dysfunction by activating the AMPK-FOXO3 cascade in cultured primary human keratinocytes. PLoS One. 2015; 10(2):e0115341.

62. Wu SB, Wu YT, Wu TP, Wei YH. Role of AMPK-mediated adaptive responses in human cells with mitochondrial dysfunction to oxidative stress. Biochim Biophys Acta. 2014; 1840(4):1331-1344. 